\section{Research Square}

Preprints are preliminary reports that have not undergone peer review.

They should not be considered conclusive, used to inform clinical practice, or referenced by the media as validated information.

\title{
Development and Validation of Assessments of Adolescent Health Literacy: A Rasch Measurement Model Approach
}

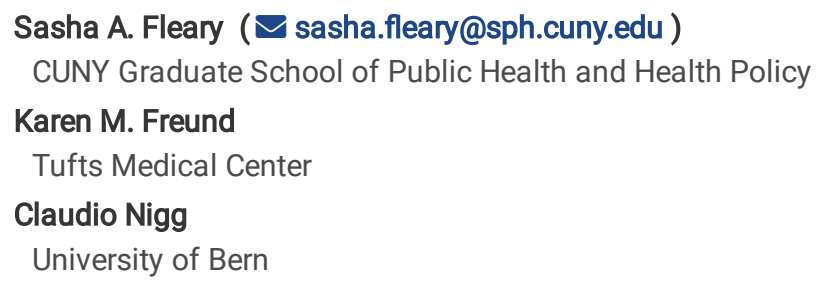

Version of Record: A version of this preprint was published at BMC Public Health on March 25th, 2022. See the published version at https://doi.org/10.1186/s12889-022-12924-4. 


\section{Abstract}

\section{Background}

Health literacy $(\mathrm{HL})$ is implicated in improved health decision-making and health promotion, and reduced racial, ethnic, and socioeconomic health disparities. Three major areas of $\mathrm{HL}$ include functional, interactive, and critical HL. HL skills develop throughout the lifespan as individuals' psychosocial and cognitive capacities develop and as they accumulate experiences with navigating health systems. Though adolescence is marked by increased involvement in health decision-making, most HL studies and measures of HL have focused on adults. Both the adult and adolescent HL literature are also limited by the paucity of validated test-based measures for assessing HL. The existing test-based validated $\mathrm{HL}$ measures for adolescents were originally designed for adults. However, adolescents are at an earlier phase of developing their HL skills (e.g., fewer experiences navigating the health system) compared to adults and measures originally designed for adults may assume prior knowledge that adolescents may lack therein underestimating adolescents' HL. This study developed and validated test-based assessments of adolescents' functional, interactive, and critical HL.

Methods

Items were generated in an iterative process: focus groups with adolescents to inform item content, cognitive interviews with adolescents and expert consultation established content and face validity of the initial items, items were revised or removed where indicated. High school students ( $\mathrm{n}=355$ ) completed a measurement battery including the revised $\mathrm{HL}$ items. The items were evaluated and validated using Rasch measurement models.

Results

The final 6-item functional, 10-item interactive, and 7-item critical HL assessments and their composite (23 items) fit their respective Rasch models. Item-level invariance was established for gender (male vs. female), age (12-15-year-olds vs. 16-18-year-olds), and ethnicity in all assessments. The assessments had good convergent validity with an established measure of functional HL and scores on the assessments were positively related to reading instructions before taking medicine and questioning the truthfulness of information found online.

\section{Conclusions}

These assessments are the first test-based measures of adolescents' interactive and critical HL, the first test-based measure of functional $\mathrm{HL}$ designed for adolescents, and first composite test-based assessment of all three major areas of HL. These assessments should be used to inform strategies for improving adolescents' HL, decision-making, and behaviors.

\section{Background}

In adults, health literacy $(\mathrm{HL})$ is implicated in a broad array of health behaviors, healthcare utilization, and outcomes including the use of prevention and emergency services, medical adherence, and interactions with providers (1-5). Further, HL has been identified as a key mediator of racial, ethnic, and socioeconomic health disparities in multiple contexts $(6,7)$. As such, $\mathrm{HL}$ has emerged as an important target for improving health decision-making and health promotion, and reducing racial, ethnic, and socioeconomic health disparities (8). In Sørenson and colleague's (8) comprehensive review of 17 definitions and 12 conceptual frameworks of individual-level HL, they summarized the concept into two dimensions: 1) core qualities of $\mathrm{HL}$ and 2) scope and reach of its applied use. Nutbeam's (9) population health-oriented definition of HL falls within the core qualities of $\mathrm{HL}$ and includes three major areas: functional $\mathrm{HL}$ (FHL; reading, writing, numeracy skills related to health information), interactive $\mathrm{HL}$ (IHL; use of health knowledge to communicate and interact with others and environment), and critical $\mathrm{HL}$ (CHL; advocacy for self and others through sociopolitical action).

Sørenson and colleagues(8) argue that HL skills develop throughout the lifespan as individuals' psychosocial and cognitive capacities develop and as they accumulate experiences with navigating health systems. Therefore, though most studies focus on adulthood, HL skills development begins earlier in the lifespan. Adolescence, in particular, is marked by increased cognitive capacity for and involvement in health decisionmaking and is therefore a salient period for developing and using HL skills (10). Yet adolescents' HL is understudied (11). Several studies have established the relationship between adolescent $\mathrm{HL}$ and health behaviors $(12,13)$. However, in their review of the literature, Fleary and colleagues (11) found that most studies exploring the relationship between adolescents' HL and health behaviors predominantly assessed FHL. In their qualitative research, Fleary and Joseph $(14,15)$ found that the $\mathrm{HL}$ skills adolescents use in their health decision-making encompass Sørenson and colleague's (8) HL definition and Nutbeam's (9) FHL, IHL, and CHL paradigm. For example, adolescents described being able to ask questions at their doctor's appointments ( $\mathrm{IHL})$ and critically analyze health information provided to them (CHL) as examples of good health decision-making skills. Therefore, it is important that these HL skills be considered in determining the role of HL in adolescents' health decisionmaking and behaviors and how best to intervene on $\mathrm{HL}$ to improve health outcomes for adolescents. 
Both the adult and adolescent HL literature are limited by the paucity of validated test-based measures for assessing the core qualities of HL outlined by Nutbeam (9). Despite the multiple definitions of HL over the years(8), most measures of HL focus primarily on FHL. For example, the Rapid Estimate of Adult Literacy in Medicine-Short Form (REALM-Short Form) is a 7-item word recognition test used in clinical settings (16). The Short-Test of Functional HL in Adults is a 40-item scale of reading and numeracy (17). The Newest Vital Sign (NVS) (18) is a 6-item measure of reading and numeracy. All of these measures were initially validated on adults. However, these is now a validated 10-item REALM-Teen short form (19) and a validation study suggesting that the NVS is valid for assessing FHL in adolescents (20). In the adolescent $\mathrm{HL}$ literature, there is a growing number of perceptions-based HL measures that include IHL and CHL (21-25). However, scales that measure perceived HL via selfreport tools may not align with actual competency and hinder actions one takes to become competent $(26,27)$. Further, using measures of perceived competencies to develop interventions may lead to misaligned programming, resulting in ineffective interventions and wasted resources. Hence, the need for test-based $\mathrm{HL}$ measures.

The existing test-based validated $\mathrm{HL}$ measures for adolescents were originally designed for adults. However, adolescents are at an earlier phase of developing their HL skills (e.g., fewer experiences with navigating the health system, cognitive and psychosocial development immature compared to adults) compared to adults and, though validated, measures originally designed for adults may assume prior knowledge that adolescents may lack therein their developmentally appropriate HL skills. For example, the NVS asks questions based on a nutrition label and prior exposure to nutrition labels may make it easier for the patient to answer the questions. However, younger adolescents in particular may score poorly on this because they lack prior experience rather than due to lack of literacy and numeracy skills for health decision-making. Therefore, it is important that HL measures for adolescents are especially designed to account for their developmental characteristics and experiences.

This study developed and validated test-based assessments of adolescents' FHL, IHL, and CHL using the Rasch measurement model. Rasch, a probabilistic model, involves testing data fit against a measurement model rather than a specific sample as is done in classical test theory (28). Analyses entail calculating the probability of a particular person responding in a particular manner to a particular item. Persons with higher ability have higher probabilities of endorsing items whereas items with higher difficulties have lower probabilities of being endorsed. Item difficulty is estimated independent of the sample and person ability is estimated independent of the items in the measure (29). Cutoff scores distinguishing levels of ability can be determined. Hence, Rasch is appropriate for developing assessments of adolescents' HL skills. We hypothesized that the final assessments would have good convergent validity with a previously validated measure of FHL. We also compared adolescents' HL test scores with their self-reported HL-related behaviors to establish criterion validity.

\section{Methods}

\section{Study Design}

A multi-phase mixed methods design was utilized to develop and validate Assessments of Adolescent Health Literacy (AAHL). As a secondary step, a composite of finalized FHL, IHL, and CHL assessments was modeled. This study was approved by the Tufts University Social Behavioral and Educational Institutional Review Board. Parent permission and adolescent assent were obtained for adolescents' participation at each phase of data collection.

\section{Measures}

\section{Demographics}

Participants indicated their age in years, gender (male, female, transgender, non-binary, other, and prefer not to answer), ethnicity (Hispanic, Latino/a or of Spanish origin), and race (Black or African American, Asian, Native America or Alaskan Native, Native Hawaiian or Other Pacific Islander, White, and Other). Participants selecting multiple races were categorized as multiracial.

\section{Newest Vital Sign}

The NVS (18), a 6-item measure of FHL with good internal consistency (Cronbach $\mathrm{a}=0.76$ ), was used to evaluate convergent validity with the AAHL. Participants responded to six reading and numeracy questions about the information on an ice cream label. Responses were scored, summed, and categorized into high likelihood of limited literacy (0-1 correct), possibility of limited literacy (2-3 correct), and adequate literacy ( $\geq 4$ correct). Summed scores were used for calculating convergent validity.

\section{HL Behaviors}

Participants indicated whether they engaged in two behaviors indicative of HL: questions the truthfulness of health information found online and reads instructions before taking medicine. These items were developed for this study based on focus groups where adolescents' described 
how they knew they used HL skills(14). These items are consistent with the scope and reach of applied use aspect of HL described by Sørenson and colleagues(8).

\section{AAHL Development}

Measurement development involved item bank development, quantitative data collection, and measurement evaluation.

\section{Item Bank Development}

Six focus groups ( 8 students/group) were conducted with $9^{\text {th }}-12^{\text {th }}$ grade high school students (Mean age $=16.49$, Standard deviation [SD]=1.35, 86.5\% girls, 35\% non-Hispanic Black, 35\% Hispanic/Latinx, 92\% free/reduced lunch eligible) to better understand adolescents' definition and operationalization of HL (full results reported elsewhere (14)). Participants also provided qualitative responses to scenarios related to $\mathrm{IHL}$ and $\mathrm{CHL}$. These focus groups were the first step to ensuring that the resulting assessments had content validity (measure adequately represents all aspects of the construct) and was developmentally appropriate (30). The responses to the scenarios included in the focus groups were content-analyzed and used to inform responses to similar scenarios in the item bank and to develop other scenarios that would be familiar to adolescents. Initial items were written by the primary author and revised after feedback from the second and third authors and doctoral-level research assistants. To establish face validity and further establish content validity, four graduate-level research assistants who completed substantial reading on $\mathrm{HL}$ and were involved in multiple $\mathrm{HL}$ projects but uninvolved in the item bank development engaged in a sorting activity to put items in three categories based on Nutbeam's (9) definitions of FHL, IHL, and CHL. Two physicians who worked with adolescents were also provided with Nutbeam's (9) definitions and the items and asked to indicate which type of HL the items belong to (if any) and provide feedback on current items and suggestions for additional items. Items were removed or revised if they were not unanimously sorted into the three categories. Items were also revised, removed, or added based on suggestions and feedback from the physicians. Next, adolescents 12-17-years-old were recruited from after-school settings to participate in cognitive interviews. Adolescents ( $\mathrm{n}=17$, Mean age=15.88 years, $S D=1.69,47 \%$ girls, $41 \%$ non-Hispanic Black, 53\% Hispanic/Latinx, 94\% free/reduced lunch eligible) participated in the cognitive interviews while they completed the item bank questions. This process further established content validity as it provided data on how adolescents interpreted the questions and their thought processes as they responded. Cognitive interviews results were used to improve (e.g., rewording questions, calibrating the difficulties of the items) or remove problematic items. Figure 1 shows the iterative changes from initial item bank development to the revised item bank used for quantitative data collection. For both focus groups and cognitive interviews, data collection was discontinued once saturation was reached.

\section{Quantitative Data Collection and Measurement Evaluation}

To refine the assessments further, the items were administered to a larger sample and Rasch measurement models were used to identify items that best fit the latent constructs of FHL, IHL, and CHL. A convenience sample of adolescents (12-18-years-old) was recruited from a local high school via flyers and classroom announcements. Data collection was coordinated with the school's head health teacher and students completed the survey during health class. Some of the health classes were required and introductory-level while others were electives for adolescents who were interested in health careers. All students present completed the surveys, however, only data from students with signed permission and assent forms were used for study purposes $(n=355)$. The survey was administered via Qualtrics on researcher-provided tablets. Students received a \$15 gift card for their participation.

\section{Statistical analyses}

Rasch measurement models were estimated in Winsteps (31) and all other analyses were conducted in SPSS 26 (32).

FHL and IHL items were analyzed using the Rasch dichotomous model. CHL items were analyzed using the the Rasch Partial Credit Model as response options were indicative of different degrees of $\mathrm{CHL}$. Key assumptions of Rasch include unidimensionality, local independence, and monotonicity of the latent trait. Unidimensionality, whether items assessed a shared latent construct, was evaluated by examining the principal components analysis of the residuals (33). If the eigenvalue of the unexplained variance in the first contrast was $<2$, then unidimensionality was assumed (34). Local independence, whether item responses were statistically independent of each other, was assessed by examining the standardized residual correlations of the items. Items with correlations $<0.50$ were considered independent (35). Monotonicity of the latent trait, whether scores were monotonically non-decreasing across the latent trait, was confirmed by monotonically ascending test characteristic curves(36).

Joint maximum likelihood estimation was used to estimate person and item parameters. Fit statistics included infit and outfit mean-squares and their standardized equivalents for both parameters. Mean-squares 0.5-1.5 indicated good fit and those $<0.5$ or 1.6-2.0 were considered unproductive for measurement but did not degrade the measure. Standardized statistics $>2$ or $<-2$ indicated significant misfit (37). Outfit statistics are most sensitive to misalignment of person responses and item difficulties. Item quality was judged by first identifying items with outfit mean-squares $>1.5$, for these items, outfit standardized statistics were evaluated and items with values $>2$ were considered for removal. 
Items with outfit $<0.5$ were less concerning (38). Items were removed iteratively starting with those with the highest mean-square outfit misfit with standardized outfit statistics $>2$. Fit statistics were recalculated and reevaluated until the items fit the model. Items with negative pointmeasure correlations were removed as these correlations indicated that the items were negatively polarized with the rest of the scale (33, 39). In addition to item misfit, person misfit was also assessed. Similar to procedures in other studies (40) and proposed by Linacre (37), tables of most misfitting responses were examined, and for each analyses, 1 round of the most misfitting responses were removed and compared with the original model. If removing the misfitting responses did not improve model fit, the original model was retained. However, when model fit was improved, final analyses were conducted on the dataset with the misfitting responses removed. Consistent with other studies using Rasch, final decisions to retain or delete items were based on both statistical findings and theoretical reasoning for the items (41). Unidimensionality, local independence, and monotonicity were examined at each iteration. See Table 1 for item difficulties and fit statistics for the final assessments.

Item-separation reliability statistics were examined to determine if the item difficulty range was sufficient with scores closer to 1 indicating good item separation. Wright (42) argued that the reliability of a test for a sample assumes symmetric ability which is rarely the case in health-related research and proposed an alternative method of calculating reliability, Wrights' sample-independent reliability statistics, to be employed once measure calibration was completed (42) in place of Winsteps' person reliability statistics. The procedures involved determining the number of strata across the scores then using this to calculate the sample-independent reliability: number of levels ${ }^{2} / 1+$ number of levels $^{2}$. Sampleindependent reliability was more appropriate for this study given that the sample was skewed in ability. Lastly, invariance of items across subpopulations were assessed by calculating uniform differential item functioning (DIF) for gender, age, and ethnicity. A minimum of 100 participants per group is required to detect DIF that is $\geq 0.5$ logits and statistically significant (43). Due to small sample sizes, age was grouped 12-15-years-old and 16-18-years-old to calculate DIF. For all DIF analyses, significance thresholds were set to $p<0.01$ to account for multiple tests. The sample size for the Rasch model calculations was appropriate as models can be estimated with $99 \%$ confidence within 0.5 logits with a minimum sample of 108 to 243 (44). For the Rasch Partial Credit Model, each response category surpassed the minimum requirement of 10 responses (45).

After finalizing the assessments, descriptive statistics were calculated. Adolescents 12-14 years were combined due to small n. Similarly, adolescents who identified as Asian, American Indian or Alaska Native/Native Hawaiian or Other Pacific Islander were combined due to small n. Convergent validity (whether two measures of constructs that should be related are related(46)) of the AAHL with an existing measure of FHL (NVS) was assessed by examining the correlations between the summed scores of both measures. Criterion validity (whether the score on one measure is related to a direct outcome of the phenomenon(47)) was assessed by modeling logistic regressions predicting HL-related behaviors from AAHL after controlling for demographics. Effect sizes were also estimated by estimating receiver operating characteristic curves and transforming the area under the curves to Cohen's $d$ using tables proposed by Salgado (48). To compare mean AAHL scores and categorizations, mean differences of each HL's assessment scores and their effect sizes were computed for categorizations.

\section{Results}

The sample consisted of 355 adolescents (Mean age=16 years, SD=1.34; 55\% girls). All but one participant chose either male or female. The largest racial group was Other ( 35\%) and $\sim 51 \%$ of the sample was non-Hispanic/Latinx. Of the subsample ( $n=200)$ that completed the NVS, $35 \%$ had a high likelihood of limited literacy and $27 \%$ had adequate literacy. See Table 2 for descriptive statistics.

\section{FHL}

The revised FHL item bank contained 12 items assessing numeracy and reading skills mainly using a cafeteria menu and over-the-counter prescription label. Six items were removed due to outfit misfit. Removal of the most misfitting person responses did not improve model fit, therefore all responses were retained. The final 6-item FHL assessment evaluated reading comprehension, reading charts, and numeracy skills (see Appendix A). Point-measure correlations for the final assessment were 0.43-0.63 suggesting high correlations with person abilities. Assumptions of unidimensionality (eigenvalue=1.4), local independence (correlations $\leq-0.31$ ), and monotonicity were met. No DIF was detected for gender, age, and ethnicity. Item separation reliability (0.97) was acceptable. The Wright sample-independent reliability statistic was 0.80 and the scores differentiated two distinct levels of performances: Emerging (scores 0-4) and Expanding FHL (scores 5-6). The Kuder-Richardson Formula 20 (KR-20) alpha was 0.63.

FHL scores (Mean=4.29, SD=1.51) differed significantly by age and NVS category. Specifically, adolescents 16-years-old had significantly higher scores than adolescents 12-14-years-old (Mean difference $=0.80, p=0.030$ ) and 18-year-olds (Mean difference $=0.98, p=0.019$ ). For NVS, adolescents with high likelihood of limited literacy had significantly lower FHL scores than adolescents with possibility of limited literacy (Mean difference $=-1.08, p<0.001$ ) and adequate literacy (Mean difference $=-1.58, p<0.001$ ). The assessment had convergent validity with the NVS $(r=0.44, p<0.001)$. Regarding criterion validity, the assessment was positively related to adolescents questioning truthfulness of health information found online (odds ratio $[\mathrm{OR}]=1.31,95 \%$ confidence interval $[\mathrm{Cl}]: 1.10,1.58, d=0.43$ ) and reading instructions before taking medicine (OR=1.31, $\mathrm{Cl}: 1.02,1.69, d=0.49)$. 
The revised IHL item bank contained 15 items and 10 items were retained for the final assessment (see Appendix B). The final assessment evaluated adolescents' skills for interacting with providers, multiple sources of contradictory information, and using knowledge to inform current behavior. Four items were removed due to high outfit statistics and one item was removed due to low point-measure correlation. Removal of the most misfitting person responses improved model fit, therefore final model estimation was done on the dataset with misfitting responses removed. Point-measure correlations for the final assessment were $0.42-0.66$. Assumptions of unidimensionality (eigenvalue=1.6), local independence (correlations $\leq 0.30$ ), and monotonicity were met. No DIF was detected for gender, age, and ethnicity. Item separation reliability (0.98) was acceptable. The Wright sample-independent reliability statistic was 0.80 and the scores differentiated two distinct levels of performances: Emerging (scores 0-5) and Expanding IHL (scores 6-10). The KR-20 alpha was 0.74.

IHL scores (Mean=8.07, SD=1.88) differed by gender and NVS category. Adolescent girls had significantly higher IHL scores than adolescent boys (Mean difference $=1.07, \mathrm{p}<0.001$ ). Adolescents with high likelihood of limited literacy had significantly lower IHL scores than adolescents with possibility of limited literacy (Mean difference $=-1.36, p<0.001$ ) and adequate literacy (Mean difference $=-1.99, p<0.001$ ). Convergent validity with the NVS was established $(r=0.43, \mathrm{p}<0.001)$. Regarding criterion validity, IHL was positively related to questioning the truthfulness of health information found online $(\mathrm{OR}=1.43, \mathrm{Cl}: 1.21,1.68, d=0.67)$ and reading instructions before taking medicine $(\mathrm{OR}=1.43, \mathrm{Cl}: 1.16,1.77$, $d=0.66)$.

\section{$\mathrm{CHL}$}

The revised $\mathrm{CHL}$ item bank contained 9 items that assessed skills for engaging in advocacy around health issues and understanding how socioeconomic barriers may impact health decisions. Seven of the 9 items were retained for the final assessment (see Appendix C). This assessment was evaluated using the Rasch Partial Credit Model. The response options were ranked from not at all CHL to collective advocacy (where applicable) skills, except for items CRHLD2 and CRHLD6 which were scored as incorrect or correct. One item was removed due to high misfit outfit mean-square statistics and a second item was removed due to extremely low point-measure correlation. Removal of the most misfitting person responses did not improve model fit, therefore all responses were retained. Point-measure correlations for the final assessment were 0.51-0.68. Assumptions of unidimensionality (eigenvalue=1.4), local independence (correlations $\leq 0.31$ ), and monotonicity were met. No DIF was detected for gender, age, and ethnicity. Item separation reliability (0.95) was acceptable. The Wright sample-independent reliability statistic was 0.80 with the scores differentiating two distinct levels of performances: Emerging (scores 0-7) and Expanding (scores 8-14) CHL. The KR-20 alpha was 0.74. Note that the scores ranged from 0-14 though only 7 items were retained. This is because with Rasch Partial Credit Models each polytomous response option has a unique score that corresponds to degree of correctness.

$\mathrm{CHL}$ scores (Mean=11.32, SD=2.76) differed significantly by gender, age, and NVS category. Adolescent girls scored significantly higher than adolescent boys (Mean difference $=0.78, p=0.013$ ). Adolescents 16-years-old scored higher than 18-year-olds $($ Mean difference $=1.86$, $\mathrm{p}=0.017$ ). Regarding the NVS, adolescents with a high likelihood of limited literacy scored significantly lower than adolescents with possibility of limited literacy (Mean difference $=-2.20, p<0.001$ ) and adequate literacy (Mean difference $=-3.40, p<0.001$ ) while adolescents with possibility of limited literacy scored lower than adolescents with adequate literacy (Mean difference $=-1.20, p=0.011$ ). NVS was significantly positively correlated with the $\mathrm{CHL}$ assessment $(r=0.52, \mathrm{p}<.01)$, therefore convergent validity was established. Regarding criterion validity, $\mathrm{CHL}$ was positively related to questioning the truthfulness of health information found online $(\mathrm{OR}=1.25, \mathrm{Cl}: 1.12,1.40, d=0.61)$ and reading instructions before taking medicine (OR=1.27, $\mathrm{Cl}: 1.10,1.46, d=0.66)$.

\section{AAHL Composite}

A Rasch Partial Credit Model was estimated to evaluate how well the final items in the FHL, IHL, and CHL assessments fit in a single model. Items FHLD3 and FHLD7 had standardized outfit statistics above 2.0, however, outfit and infit mean-square fit statistics were in the acceptable range so no further action was required. Point-measure correlations were $0.33-0.62$. Assumptions of unidimensionality (eigenvalue=1.8), local independence (correlations $\leq 0.42$ ), and monotonicity were met. No DIF was detected for gender, age, and ethnicity. Item separation reliability (0.97) was acceptable. Wright sample-independent reliability statistic was 0.94 with the scores differentiating four levels of performances: Emerging (0-10), Expanding (11-19), Lower Bridging (20-27), and Upper Bridging (28-30). The KR-20 alpha was 0.91.

AAHL Composite scores (Mean=23.92, SD=5.04) differed significantly by gender, age, and NVS category. Adolescent girls scored significantly higher than adolescent boys (Mean difference $=2.19, \mathrm{p}<0.001$ ). Adolescents 16-years-old had higher scores than 18-year-olds (Mean difference $=3.13, p=0.044$ ). Regarding the NVS, adolescents with a high likelihood of limited literacy scored significantly lower than adolescents with possibility of limited literacy (Mean difference $=-4.71, p<0.001$ ) and adequate literacy (Mean difference $=-6.94, p<0.001$ ) while adolescents with possibility of limited literacy scored lower than adolescents with adequate literacy (Mean difference $=-2.24, p=0.013$ ). The NVS was positively correlated with the AAHL Composite $(0.56, p<.01)$ establishing convergent validity. Regarding criterion validity, composite scores were positively related to questioning truthfulness of health information found online $(\mathrm{OR}=1.15, \mathrm{Cl}: 1.08,1.22, d=0.73)$ and reading instructions before taking 
medicine $(\mathrm{OR}=1.15, \mathrm{Cl}=1.06,1.24, d=0.79)$. Similar to $\mathrm{CHL}$, score ranges were large as a Rasch Partial Credit Model was estimated and each polytomous response option had a unique score that corresponded to degree of correctness.

\section{Comparing $\mathrm{FHL}, \mathrm{IHL}$, and $\mathrm{CHL}$ Scores}

Adolescents categorized as having Expanding $\mathrm{FHL}$ had higher $\mathrm{IHL}(d=0.68), \mathrm{CHL}(d=0.77)$, and composite $\mathrm{HL}(d=1.14)$ than those categorized as Emerging. Adolescents categorized as having Expanding IHL had higher FHL $(d=1.38), \mathrm{CHL}(d=1.85)$, and composite $\mathrm{HL}(d=2.35)$ than those categorized as Emerging. Adolescents categorized as having Expanding $\mathrm{CHL}$ had higher $\mathrm{FHL}(d=1.44)$, IHL $(d=1.78)$, and composite $\mathrm{HL}(d=2.40)$ than those categorized as Emerging.

\section{Discussion}

This study aimed to develop and validate test-based assessments of adolescents' FHL, IHL, and CHL. Face and initial content validity were established using focus groups, expert review, and cognitive interviews in the early phases of the study. The final assessments fit their respective Rasch models and met the key Rasch assumptions of unidimensionality, local independence, and monotonicity. Key Rasch assumptions were also met when all items across the three assessments were entered into a single Rasch model. Each assessment had good convergent and criterion validity.

Nutbeam (9) proposed that FHL, IHL, and CHL are nested skills with each requiring a more complex/sophisticated skill set. In our item set, the difficulty level on the FHL items were relatively higher than those on the $\mathrm{IHL}$ and $\mathrm{CHL}$. This is not surprising as measures of FHL assess numerical and reading skills which are highly academic in content while the other measures assess social, interpersonal, and "know how" that one can acquire via opportunities for modeling, scaffolding, and practice. This aligns with the argument that HL is a type of cultural health capital (49). When our FHL assessment and the NVS were included in a single Rasch model, 5 of the 6 NVS items were more difficult than the highest difficulty item on our FHL assessment. Therefore, the FHL assessment performing differently than what is proposed theoretically is less likely to be due to our assessment's difficulty. We propose that there are qualitative differences in how HL skills may be acquired and these differences may explain why FHL may be a more difficult skillset than IHL and CHL during adolescence. Test-based measures of IHL and CHL for adults to determine if the same patterns of difficulty are found in adults are needed.

Relatedly, we chose a cafeteria menu and over-the-counter medication label rather than a nutrition facts label for the initial FHL assessment as we expected these would be more familiar to adolescents. In our experience using the NVS with younger adolescents (i.e., 13-14-year-olds), being presented with the nutrition label is overwhelming and anxiety-provoking for adolescents likely due to unfamiliarity with reading nutrition labels. Given that HL skills develop through experience (8) and that adolescents may have more experience reading a cafeteria menu than a nutrition label, we expected the familiarity of the menu would be more conducive to adolescents excelling at demonstrating their skills. This may also explain why adolescents performed better on the FHL measure when compared to the NVS and provides support for the need for HL measures developed specifically for adolescents rather than validating adult measures in adolescent samples. In cognitive interviews, the cafeteria menu tested slightly better than the over-the-counter medication label. However, responses on the over-the-counter medication label were inconsistent when evaluated in the Rasch model. We propose that exposure to cafeteria menus and over-the-counter medication labels differ with fewer adolescents having sufficient exposure to medication labels (compared to cafeteria menus) to not be overwhelmed when their reading and numeracy skills are tested using these labels.

Of Nutbeam's (9) three HL concepts, CHL is the most complicated to operationalize. Sykes and colleagues (50) conducted a study on the conceptualization of $\mathrm{CHL}$ across discipline, time, place, and multiple types of users and found that definitions ranged from emphasizing higher order cognitive skills to empowerment and critical engagement to affect sociopolitical change. We attempted to represent the range of definitions from individual cognitive skills to collective advocacy to affect community health in our response options. Hence, the use of Rasch Partial Credit Model for the $\mathrm{CHL}$ assessment and the ranking of the options from not at all HL to collective advocacy (where applicable). This made for a more accurate assessment of the skill than would be estimated if the responses were dichotomized into correct and incorrect. Further, use of focus groups to elicit community health topics important to adolescents and modes of advocacy in which adolescents engaged or wish to engage allowed for a robust $\mathrm{CHL}$ assessment that was relatable and relevant to adolescents' lived experiences.

The effect sizes for predicting $\mathrm{HL}$ behaviors from $\mathrm{FHL}$, IHL, and $\mathrm{CHL}$ ranged from $0.43-0.67$. These effect sizes suggest that the final objective assessments have utility in predicting behavior and for assessing HL skills necessary for engaging in applied HL. The Composite score was a stronger indicator of the $\mathrm{HL}$ behaviors (0.73-0.79) suggesting that assessing these three types of $\mathrm{HL}$ together is a better indicator of $\mathrm{HL}$ skills than FHL alone as is common in both the adult and adolescent literature $(2,5,11)$. Our analyses reinforce Sørenson et al.'s(8) definition of $\mathrm{HL}$ as our assessments tested "do you have the skills?" and the relationship between these skills and applied use was confirmed through acceptable effect sizes. 
To date, test-based HL measures that have been validated in adolescents were all initially developed for adult populations. A major strength of this study is that the adolescent $\mathrm{HL}$ assessments were designed with consideration and inclusion of adolescents' lived experiences. This study also provides the first test-based measures for IHL and CHL as well as core qualities of HL (AAHL Composite) as described by Sørenson and colleagues (8) across the adolescent and adult HL literature. Though these measures were developed for and in collaboration with adolescents, the items in the measures are relevant to both adolescent and adult populations. Therefore, future studies should assess the validity of these assessments with adult samples especially given the lack of test-based measures of core qualities of HL in the literature.

This study is not without limitations. First, the sample ability was skewed due to adolescents being enrolled in health classes with some having interest in health careers. However, the sample-independent reliability corrected for this and allowed for identification of multiple strata of the constructs. Future studies should replicate the validity of the assessments in a sample of more diverse person ability. Second, though the sample size was sufficient for conducting the Rasch analysis, it was insufficient for calculating DIF for each age and for race. Despite this, the racial diversity of participants throughout all phases of the measurement development is a unique strength of the study. Future studies should be amply powered to explore item invariance for race and other demographic variables of interest such as parent education and family household income. These assessments should also be employed in longitudinal designs to determine their predictive validity and ability to detect change.

These FHL, IHL, and $\mathrm{CHL}$ assessments and their composite have utility in multiple settings. In school settings, health teachers may use students' scores and categorization to identify curricular needs as well as to assess proficiency before and after relevant coursework. In medical settings, the assessments may be used to identify adolescents who may need additional support navigating their health care especially in cases where adolescents have the option to see providers without parental consent/attendance. This is particularly important as most states have minor consent laws for sexual health (adolescents may see providers about sexual-health related issues without parental consent) and some states have minor consent laws beyond sexual health $(51,52)$. Interventionists may also use the assessments to identify $\mathrm{HL}$ intervention needs for adolescents as well as to ensure that their non-HL interventions are effective across the range of $\mathrm{HL}$ skills.

\section{Conclusion}

This study aimed to develop test-based measures of adolescents' FHL, IHL, and CHL. The finalized assessments met all the assumptions of Rasch, and had good model fit, and convergent and criterion validity. These assessments are the first test-based measures of adolescents' IHL and $\mathrm{CHL}$, the first test-based measure of FHL designed specifically for adolescents, and first composite test-based assessment of the core qualities of $\mathrm{HL}$. These assessments have utility in multiple settings and will contribute significantly to how these constructs are studied and intervened on in future studies and programs.

\section{Abbreviations}

AAHL: Assessments of adolescent health literacy

CHL: Critical health literacy

Cl: Confidence interval

DIF: Differential item function

FHL: Functional health literacy

HL: Health literacy

IHL Interactive health literacy

KR-20: Kuder-Richardson Formula 20

NVS: Newest vital sign

OR: Odds ratio

\section{Declarations}

Ethics approval and consent to participate 
This study was approved by the Tufts University Social Behavioral and Educational Institutional Review Board. All methods were performed in accordance to the approved protocol and relevant guidelines and regulations for human participants research. Parent permission (parent version of informed consent for adolescent participation) and adolescent assent (adolescent version of informed consent given that adolescents cannot legally provide informed consent) were obtained for adolescents' participation at each phase of data collection.

\section{Consent for publication}

Not applicable.

\section{Availability of data and materials}

The dataset analysed during the current study are not publicly available given that it is a relatively small dataset of a protected population but are available from the corresponding author on reasonable request.

\section{Competing interests}

The authors declare that they have no competing interests.

\section{Funding}

This work was supported by the National Institute of Health [grant numbers 1K12HD092535, L30DK126209]. The funding agencies had no role in the design of the study and collection, analysis, and interpretation of data and in writing the manuscript.

\section{Author contributions}

SAF designed the study with contributions from KF and CN. SAF collected the data. SAF analyzed and interpreted the data with contributions from KF and CN. SAF drafted the manuscript and KF and CN revised the manuscript. All authors have approved the submitted version and agree to be personally accountable for their contributions. All authors agree to ensure that questions related to the accuracy or integrity of any part of the work, even ones in which the author was not personally involved, are appropriately investigated, resolved, and the resolution documented in the literature.

\section{Acknowledgments}

The author wishes to acknowledge Patrece Joseph and Carolina Goncalves and other research assistants affiliated with the Child Health Equity Research Lab for their contributions to the project. The authors also wish to thank community collaborators and all research participants.

\section{References}

1. Zhang NJ, Terry A, McHorney CA. Impact of health literacy on medication adherence: a systematic review and meta-analysis. Annals of Pharmacotherapy. 2014;48(6):741-51.

2. Miller TA. Health literacy and adherence to medical treatment in chronic and acute illness: A meta-analysis. Patient education and counseling. 2016;99(7):1079-86.

3. Aaby A, Friis K, Christensen B, Rowlands G, Maindal HT. Health literacy is associated with health behaviour and self-reported health: A large population-based study in individuals with cardiovascular disease. European journal of preventive cardiology. 2017;24(17):1880-8.

4. Morrison AK, Schapira MM, Gorelick MH, Hoffmann RG, Brousseau DC. Low caregiver health literacy is associated with higher pediatric emergency department use and nonurgent visits. Academic pediatrics. 2014;14(3):309-14.

5. Berkman ND, Sheridan SL, Donahue KE, Halpern DJ, Crotty K. Low health literacy and health outcomes: an updated systematic review. Annals of internal medicine. 2011;155(2):97-107.

6. Stormacq C, Van den Broucke S, Wosinski J. Does health literacy mediate the relationship between socioeconomic status and health disparities? Integrative review. Health promotion international. 2019;34(5):e1-e17.

7. Mantwill S, Monestel-Umaña S, Schulz PJ. The relationship between health literacy and health disparities: a systematic review. PLoS One. 2015;10(12):e0145455.

8. Sørenson K, Van den Broucke S, Fullam J, Doyle G, Pelikan J, Slonska Z, et al. Health literacy and public health: a systematic review and integration of definitions and models. BMC public health. 2012;12(80):1-13. 
9. Nutbeam D. The evolving concept of health literacy. Social science \& medicine. 2008;67(12):2072-8.

10. Manganello JA. Health literacy and adolescents: a framework and agenda for future research. Health Education Research. 2008;23(5):840-7.

11. Fleary SA, Joseph P, Pappagianopoulos J. Adolescent Health Literacy and Health Behaviors: A Systematic Review. Journal of Adolescence. 2018;62:116-27.

12. Park A, Eckert TL, Zaso MJ, Scott-Sheldon LA, Vanable PA, Carey KB, et al. Associations between health literacy and health behaviors among urban high school students. Journal of School Health. 2017;87(12):885-93.

13. Chisolm DJ, Manganello JA, Kelleher KJ, Marshal MP. Health literacy, alcohol expectancies, and alcohol use behaviors in teens. Patient education and counseling. 2014;97(2):291-6.

14. Fleary SA, Joseph P. Adolescents' Health Literacy and Decision-making: A Qualitative Study. American journal of health behavior. 2020;44(4):392-408.

15. Joseph P, Fleary SA. The Way You Interpret Health": Adolescent Definitions and Perceptions of Health Literacy. Journal of School Health. 2021.

16. Arozullah AM, Yarnold PR, Bennett CL, Soltysik RC, Wolf MS, Ferreira RM, et al. Development and validation of a short-form, rapid estimate of adult literacy in medicine. Medical care. 2007:1026-33.

17. Baker DW, Williams MV, Parker RM, Gazmararian JA, Nurss J. Development of a brief test to measure functional health literacy. Patient Education and Counseling. 1999;38(1):33-42.

18. Weiss BD, Mays MZ, Martz W, Castro KM, DeWalt DA, Pignone MP, et al. Quick assessment of literacy in primary care: the newest vital sign. The Annals of Family Medicine. 2005;3(6):514-22.

19. Manganello JA, Colvin KF, Chisolm DJ, Arnold C, Hancock J, Davis T. Validation of the Rapid Estimate for Adolescent Literacy in Medicine Short Form (REALM-TeenS). Pediatrics. 2017;139(5):e20163286.

20. Warsh J, Chari R, Badaczewski A, Hossain J, Sharif I. Can the Newest Vital Sign Be Used to Assess Health Literacy in Children and Adolescents? Clinical Pediatrics. 2013;53(2):141-4.

21. Manganello JA, DeVellis RF, Davis TC, Schottler-Thal C. Development of the Health Literacy Assessment Scale for Adolescents (HAS-A). Journal of Communication in Healthcare. 2015;8(3):172-84.

22. Ghanbari S, Ramezankhani A, Montazeri A, Mehrabi Y. Health Literacy Measure for Adolescents (HELMA): Development and Psychometric Properties. PloS one. 2016;11(2):e0149202.

23. Bollweg TM, Okan O, Freţian AM, Bröder J, Domanska OM, Jordan S, et al. Adapting the European Health Literacy Survey Questionnaire for Fourth-Grade Students in Germany: Validation and Psychometric Analysis. HLRP: Health Literacy Research and Practice. 2020;4(3):e144e59.

24. Paakkari O, Torppa M, Villberg J, Kannas L, Paakkari L. Subjective health literacy among school-aged children. Health Education. 2018.

25. Sarkar U, Schillinger D, López A, Sudore R. Validation of Self-Reported Health Literacy Questions Among Diverse English and SpanishSpeaking Populations. Journal of General Internal Medicine. 2011;26(3):265-71.

26. Butler R. Determinants of help seeking: Relations between perceived reasons for classroom help-avoidance and help-seeking behaviors in an experimental context. Journal of Educational Psychology. 1998;90(4):630.

27. Nelson-Le Gall S, Kratzer L, Jones E, DeCooke P. Children's self-assessment of performance and task-related help seeking. Journal of Experimental Child Psychology. 1990;49(2):245-63.

28. Wolfe EW, Smith Jr EV. Instrument development tools and activities for measure validation using Rasch models: Part I-instrument development tools. Journal of applied measurement. 2007.

29. Wright B, Panchapakesan N. A procedure for sample-free item analysis. Educational and Psychological measurement. 1969;29(1):23-48. 
30. Vogt DS, King DW, King LA. Focus groups in psychological assessment: enhancing content validity by consulting members of the target population. Psychological assessment. 2004;16(3):231.

31. Linacre JM. Winsteps ${ }^{\circledR}$ Rasch measurement computer program. Beaverton, Orego: Winsteps.com; 2021.

32. IBM Corp. IBM SPSS Statistics for Windows, Version 26.0. Armonk, NY: IBM Corp; 2019.

33. Wolfe EW, Smith Jr EV. Instrument development tools and activities for measure validation using Rasch models: part II-validation activities. Journal of applied measurement. 2007;8(2):204-34.

34. Raîche G. Critical eigenvalue sizes in standardized residual principal components analysis. Rasch measurement transactions. 2005;19(1):1012.

35. Andrich D, Kreiner S. Quantifying response dependence between two dichotomous items using the Rasch model. Applied Psychological Measurement. 2010;34(3):181-92.

36. Kang $\mathrm{H}-\mathrm{A}$, Su $\mathrm{Y}-\mathrm{H}$, Chang $\mathrm{H}-\mathrm{H}$. A note on monotonicity of item response functions for ordered polytomous item response theory models. British Journal of Mathematical and Statistical Psychology. 2018;71(3):523-35.

37. Linacre JM. What do infit and outfit, mean-square and standardized mean. Rasch measurement transactions. 2002;16(2):878.

38. Wright B, Linacre J, Gustafson J, Martin-Löf P. Reasonable mean-square fit values. Rasch Measurement Transactions, 8, 370. Statistická analýza. 1994:0-60.

39. Frisbie DA. Measurement 101: Some fundamentals revisited. Educational Measurement: Issues and Practice. 2005;24(3):21-8.

40. Rees G, Xie J, Fenwick EK, Sturrock BA, Finger R, Rogers SL, et al. Association between diabetes-related eye complications and symptoms of anxiety and depression. JAMA ophthalmology. 2016;134(9):1007-14.

41. Finlayson ML, Peterson EW, Fujimoto KA, Plow MA. Rasch validation of the falls prevention strategies survey. Archives of physical medicine and rehabilitation. 2009;90(12):2039-46.

42. Wright BD. Separation, reliability and skewed distributions: statistically different levels of performance. Rasch Meas Trans. $2001 ; 14(4): 786$.

43. Linacre JM. DIF Sample Size Nomogram. Rasch Meas Trans. 2013;26(4):1392-402.

44. Linacre J. Sample size and item calibration stability. Rasch Mes Trans. 1994;7:328.

45. Linacre JM. Optimizing rating scale category effectiveness. Journal of applied measurement. 2002;3(1):85-106.

46. Campbell DT, Fiske DW. Convergent and discriminant validation by the multitrait-multimethod matrix. Psychological bulletin. 1959;56(2):81.

47. McDonald MP. Validity, Data Sources. In: Kempf-Leonard K, editor. Encyclopedia of Social Measurement. New York: Elsevier; 2005. p. 939-48.

48. Salgado JF. Transforming the area under the normal curve (AUC) into Cohen'sd, Pearson's rpb, odds-ratio, and natural log odds-ratio: two conversion tables. European Journal of Psychology Applied to Legal Context. 2018;10(1):35-47.

49. Shim JK. Cultural health capital a theoretical approach to understanding health care interactions and the dynamics of unequal treatment. Journal of Health and Social Behavior. 2010;51(1):1-15.

50. Sykes S, Wills J, Rowlands G, Popple K. Understanding critical health literacy: a concept analysis. BMC public health. 2013;13(1):1-10.

51. Coleman DL, Rosoff PM. The legal authority of mature minors to consent to general medical treatment. Pediatrics. 2013;131(4):786-93.

52. Simmons M, Shalwitz J, Pollock S. Understanding Confidentiality and Minor Consent in California: An Adolescent Provider Toolkit. San Francisco, CA: Adolescent Health Working Group, San Francisco Health Plan; 2002.

\section{Tables}


Table 1. Rasch item difficulties and fit statistics.

Individual

Composite

\begin{tabular}{|c|c|c|c|c|c|c|c|c|c|c|}
\hline Item & Difficulty & SE & Outfit MNSQ & Outfit ZSTD & PMC & Difficulty & SE & Outfit MNSQ & Outfit ZSTD & PMC \\
\hline \multicolumn{11}{|c|}{ Functional HL } \\
\hline FHLD7 & 0.98 & 0.14 & 1.14 & 1.6 & 0.59 & 1.43 & 0.13 & 1.21 & 2.4 & 0.44 \\
\hline FHLD5 & 0.73 & 0.14 & 0.94 & -0.7 & 0.63 & 1.21 & 0.13 & 1.00 & 0.1 & 0.50 \\
\hline FHLD3 & 0.62 & 0.14 & 1.12 & 1.5 & 0.57 & 1.12 & 0.13 & 1.42 & 4.2 & 0.37 \\
\hline FHLD6 & -0.12 & 0.15 & 0.81 & -2.0 & 0.62 & 0.47 & 0.14 & 0.92 & -0.6 & 0.47 \\
\hline FHLD4 & -0.94 & 0.17 & 0.82 & -1.1 & 0.57 & -0.40 & 0.17 & 0.72 & -1.5 & 0.44 \\
\hline FHLD2 & -1.28 & 0.19 & 1.26 & 1.3 & 0.43 & -0.92 & 0.20 & 0.68 & -1.3 & 0.39 \\
\hline \multicolumn{11}{|c|}{ Interactive HL } \\
\hline ICHLD13 & 2.91 & 0.16 & 1.31 & 1.6 & 0.62 & 1.99 & 0.13 & 1.15 & 1.7 & 0.45 \\
\hline ICHLD18 & 2.02 & 0.15 & 0.98 & -0.1 & 0.66 & 1.36 & 0.13 & 1.01 & 0.1 & 0.50 \\
\hline ICHLD14 & 0.64 & 0.16 & 1.03 & -0.2 & 0.59 & 0.23 & 0.15 & 0.75 & -1.9 & 0.50 \\
\hline ICHLD17 & 0.35 & 0.17 & 0.93 & -0.4 & 0.58 & -0.02 & 0.16 & 0.78 & -1.4 & 0.51 \\
\hline ICHLD8 & -0.02 & 0.18 & 0.98 & -0.1 & 0.53 & -0.32 & 0.17 & 0.80 & -1.0 & 0.43 \\
\hline ICHLD16 & -0.26 & 0.19 & 0.87 & -0.6 & 0.48 & -0.53 & 0.18 & 0.85 & -0.6 & 0.36 \\
\hline ICHLD6 & -0.50 & 0.20 & 1.06 & 0.3 & 0.42 & -0.92 & 0.20 & 0.81 & -0.6 & 0.34 \\
\hline ICHLD9 & -1.21 & 0.24 & 0.31 & -2.6 & 0.53 & -1.28 & 0.22 & 0.36 & -2.5 & 0.45 \\
\hline ICHLD5 & -1.87 & 0.29 & 0.32 & -1.7 & 0.42 & -1.92 & 0.28 & 0.25 & -2.2 & 0.38 \\
\hline ICHLD15 & -2.05 & 0.30 & 0.29 & -1.7 & 0.42 & -2.02 & 0.29 & 0.32 & -1.7 & 0.33 \\
\hline \multicolumn{11}{|l|}{ Critical HL } \\
\hline CRHLD6 & 0.81 & 0.14 & 1.16 & 1.9 & 0.52 & 0.81 & 0.14 & 0.98 & -0.2 & 0.49 \\
\hline CRHLD2 & 0.47 & 0.15 & 1.03 & 0.4 & 0.51 & 0.50 & 0.14 & 0.99 & 0.0 & 0.47 \\
\hline CRHLPC11 & 0.36 & 0.10 & 1.11 & 0.7 & 0.58 & 0.42 & 0.10 & 1.40 & 1.7 & 0.47 \\
\hline CRHLP4 & -0.04 & 0.10 & 0.97 & -0.3 & 0.68 & 0.06 & 0.09 & 1.09 & 1.0 & 0.58 \\
\hline CRHLP5 & -0.05 & 0.09 & 0.91 & -0.7 & 0.68 & 0.05 & 0.09 & 0.88 & -0.8 & 0.62 \\
\hline CRHLP7 & -0.65 & 0.13 & 0.84 & -1.1 & 0.59 & -0.57 & 0.13 & 0.71 & -1.7 & 0.54 \\
\hline ICCHLP3R & -0.89 & 0.13 & 0.85 & -0.9 & 0.54 & -0.80 & 0.13 & 0.75 & -1.2 & 0.51 \\
\hline
\end{tabular}

Notes. $\mathrm{HL}=$ health literacy; $\mathrm{MNSQ}=$ Mean-square; $\mathrm{PMC}=$ point-measure correlation; $\mathrm{SE}$ = standard error; ZSTD = standardized statistic

Table 2. Descriptives of the sample 


\begin{tabular}{|c|c|c|c|c|c|c|c|c|c|}
\hline Variable & (n)\% & $\begin{array}{l}\text { FHL } \\
\text { M(SD) }\end{array}$ & $F$ & $\begin{array}{l}\text { IHL } \\
\text { M(SD) }\end{array}$ & $\mathbf{F}$ & $\begin{array}{l}\mathrm{CHL} \\
\mathrm{M}(\mathrm{SD})\end{array}$ & $\mathbf{F}$ & $\begin{array}{l}\text { Composite } \\
\text { M (SD) }\end{array}$ & $\mathbf{F}$ \\
\hline Gender & & & 1.47 & & $25.94^{\star \star \star}$ & & $6.30 *$ & & $13.93^{\star \star \star}$ \\
\hline Boys & $136(45.2)$ & $4.24(1.58)$ & & $7.54(2.12)$ & & $10.99(2.79)$ & & $22.84(5.53)$ & \\
\hline Girls & $165(54.8)$ & $4.45(1.37)$ & & $8.61(1.43)$ & & 11.78(2.53) & & $25.03(4.25)$ & \\
\hline Missing & 54 & & & & & & & & \\
\hline Age (years) & & & $3.51^{\star \star}$ & & 1.46 & & $2.60 *$ & & $2.56^{\star}$ \\
\hline $12-14$ & $57(18.7)$ & $4.09(1.60)$ & & 7.77(2.15) & & $11.33(2.77)$ & & $23.22(5.58)$ & \\
\hline 15 & $50(16.4)$ & $4.49(1.37)$ & & 8.33(1.64) & & $11.60(2.43)$ & & $24.47(4.48)$ & \\
\hline 16 & $63(20.7)$ & $4.89(1.10)$ & & $8.44(1.64)$ & & $12.05(2.17)$ & & $25.36(3.85)$ & \\
\hline 17 & 101(33.1) & $4.29(1.51)$ & & $8.13(1.84)$ & & $11.32(2.71)$ & & $23.99(5.03)$ & \\
\hline 18 & $34(11.1)$ & $3.90(1.46)$ & & 7.72(2.07) & & $10.19(3.64)$ & & $22.23(6.09)$ & \\
\hline Missing & 50 & & & & & & & & \\
\hline Hispanic/Latinx & & & 0.71 & & 1.40 & & 0.05 & & 0.46 \\
\hline Yes & $150(50.5)$ & $4.30(1.49)$ & & $8.27(1.62)$ & & $11.50(2.63)$ & & $24.33(4.52)$ & \\
\hline No & $147(49.5)$ & $4.44(1.44)$ & & $8.01(1.97)$ & & $11.43(2.66)$ & & $23.89(5.15)$ & \\
\hline Missing & 58 & & & & & & & & \\
\hline Race & & & 0.78 & & 0.99 & & 0.86 & & 1.31 \\
\hline ANAANNHOPI & $24(8.6)$ & $4.13(1.46)$ & & $7.58(2.90)$ & & $10.79(3.71)$ & & $22.30(7.33)$ & \\
\hline Black & 61(21.9) & $4.35(1.51)$ & & 7.95(1.96) & & $11.22(2.73)$ & & $23.52(5.18)$ & \\
\hline White & $66(23.7)$ & $4.61(1.50)$ & & $8.14(1.77)$ & & $11.63(2.50)$ & & $24.52(5.08)$ & \\
\hline Multiracial & $31(11.1)$ & $4.57(1.36)$ & & $8.43(1.71)$ & & $11.93(2.92)$ & & $24.96(5.02)$ & \\
\hline Other $^{\mathrm{a}}$ & $97(34.8)$ & $4.28(1.51)$ & & $8.28(1.52)$ & & $11.58(2.28)$ & & $24.39(4.05)$ & \\
\hline Missing & 76 & & & & & & & & \\
\hline $\begin{array}{l}\text { Newest Vital } \\
\text { Sign }\end{array}$ & & & $20.53^{\star \star \star}$ & & $23.71 * \star \star$ & & $36.10 * \star \star$ & & $41.54^{\star \star \star}$ \\
\hline $\begin{array}{l}\text { High } \\
\text { likelihood }\end{array}$ & $70(35)$ & $3.54(1.73)$ & & $7.03(2.15)$ & & $9.88(3.16)$ & & $20.51(4.15)$ & \\
\hline $\begin{array}{l}\text { limited } \\
\text { literacy }\end{array}$ & & & & & & & & & \\
\hline $\begin{array}{l}\text { Possibility of } \\
\text { limited } \\
\text { literacy }\end{array}$ & $76(21.4)$ & $4.61(1.15)$ & & $8.39(1.41)$ & & $12.08(1.85)$ & & $25.21(3.01)$ & \\
\hline $\begin{array}{l}\text { Adequate } \\
\text { literacy }\end{array}$ & $54(27)$ & $5.12(1.20)$ & & $9.02(1.10)$ & & $13.28(1.09)$ & & $27.45(2.15)$ & \\
\hline Missing & 155 & & & & & & & & \\
\hline Functional HL & & & 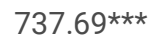 & & $39.62^{\star \star \star}$ & & $55.53^{\star \star \star}$ & & $141.63^{\star \star \star}$ \\
\hline Emerging & $155(47.7)$ & 2.97(1.09) & & $7.40(2.14)$ & & $10.20(3.15)$ & & $20.81(5.34)$ & \\
\hline Expanding & $170(52.3)$ & $5.49(0.50)$ & & $8.68(1.40)$ & & $12.35(1.84)$ & & $26.54(2.80)$ & \\
\hline Missing & 30 & & & & & & & & \\
\hline Interactive HL & & & $77.89 * * *$ & & $398.16^{\star \star \star}$ & & $170.95^{\star \star \star}$ & & $372.35^{\star \star \star}$ \\
\hline Emerging & $34(10.8)$ & $2.53(1.50)$ & & $4.03(1.09)$ & & $6.88(2.37)$ & & $13.44(3.52)$ & \\
\hline
\end{tabular}




\begin{tabular}{|c|c|c|c|c|c|c|c|c|c|}
\hline Expanding & 281(89.2) & $4.60(1.27)$ & & $8.56(1.27)$ & & 11.99(2.11) & & 25.26(3.34) & \\
\hline Missing & 40 & & & & & & & & \\
\hline Critical HL & & & 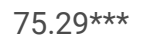 & & $117.61^{\star \star \star}$ & & 406.78 & & 285.14 \\
\hline Emerging & $35(10.9)$ & $2.36(1.56)$ & & $5.07(2.05)$ & & $5.43(1.72)$ & & 12.96(3.53) & \\
\hline Expanding & 285(89.1) & $4.54(1.34)$ & & $8.42(1.55)$ & & 12.05(1.84) & & 25.05(3.61) & \\
\hline Missing & 35 & & & & & & & & \\
\hline $\begin{array}{l}\text { AAHL } \\
\text { Composite }\end{array}$ & & & $78.04^{\star \star \star}$ & & $166.40^{\star \star \star}$ & & $225.44^{\star \star \star}$ & & $619.49 * \star \star$ \\
\hline Emerging & $7(2.3)$ & $1.71(1.11)$ & & $3.00(1.15)$ & & $4.00(2.16)$ & & $8.71(1.38)$ & \\
\hline Expanding & $44(14.7)$ & $2.70(1.39)$ & & $5.43(1.69)$ & & 7.36(1.73) & & $15.50(2.57)$ & \\
\hline $\begin{array}{l}\text { Lower } \\
\text { Bridging }\end{array}$ & $175(58.3)$ & $4.42(1.18)$ & & 8.33(1.19) & & 11.87(1.63) & & $24.61(2.01)$ & \\
\hline Upper Bridging & $74(24.7)$ & $5.57(0.53)$ & & $9.64(0.56)$ & & $13.54(0.65)$ & & 28.74(0.79) & \\
\hline
\end{tabular}

ANAANNHOPI = Asian, Native American/Alaskan Native, Native Hawaiian/Other Pacific Islander; $\mathrm{CHL}=$ critical health literacy; FHL = functional health literacy; $\mathrm{HL}=$ health literacy; $\mathrm{IHL}=$ interactive health literacy; $\mathrm{M}=$ mean; $\mathrm{SD}=$ standard deviation

a 70 adolescents who identified as Other indicated they were Hispanic/Latinx; ${ }^{\star} p<0.05 ;{ }^{* *} p<0.01 ; * \star * p<0.001$

\section{Figures}
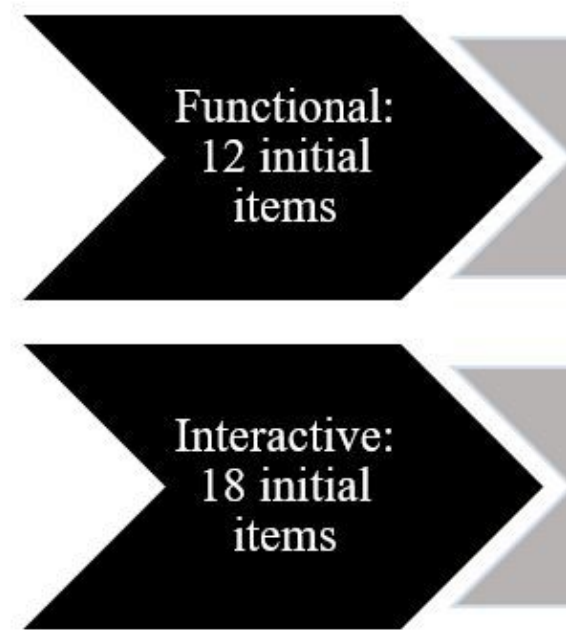

Interactive:

18 initial items

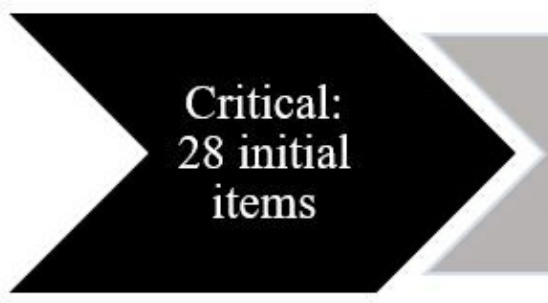

SORT/EXPERT

REVIEW

Retained $=10$;

Revised $=2$; Removed $=0$;

Added $=0 ;$ Total $=12$
COGNITIVE

INTERVIEWS

Retained $=0$; Revised $=2$;

Removed $=0$; Added $=0$;

Total $=12$

\section{Figure 1}

Illustration of iterative item bank revisions prior to large scale quantitative data collection

\section{Supplementary Files}

This is a list of supplementary files associated with this preprint. Click to download. 
- AppendixIIIHL.docx

- AppendixIFHL.docx

- AppendixIIICHL.docx

Page 15/15 